\title{
Viewing fat in zebrafish to study metabolism
}

A new technique allows scientists to visualize lipid metabolism in live zebrafish and to investigate aspects of lipid absorption that, until now, have remained poorly understood. The small intestine is the primary site of dietary lipid absorption in many vertebrates. The precise balance of nutrients, microorganisms and substances in the intestine is difficult to reproduce outside of a living organism, hindering study of the processes by which the body digests and absorbs dietary lipids in order to take in critical nutrients.

Steven A. Farber and his team at the Carnegie Institution for Science (Baltimore, MD) developed a strategy for using fluorescence to visualize lipids and relevant proteins within the cells of the small intestines of live zebrafish larvae, which are transparent, in order to observe fat and cholesterol absorption. "Historically, the zebrafish has been used in the field of embryology and development and we felt that it had been underutilized for studies of whole-animal physiology,"

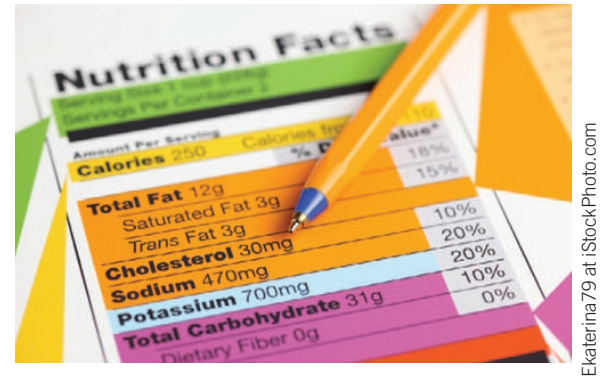

Farber said in a press release. "Using the zebrafish in this novel way allowed us to be the first to observe cholesterol absorption in a living vertebrate system."

Cholesterol and fatty acids are absorbed by intestinal cells called enterocytes. Newly absorbed fatty acids are either exported as lipoproteins or stored as fat droplets, but scientists know little about how the fate of fatty acids is determined. By studying fluorescent lipids in the zebrafish, the researchers were able to determine that fatty acid conversion depends on the level of fatty acids in the intestine: when fatty acid levels are high, fat storage in the form of lipid droplets is favored (Chem. Biol. 19, 1-13; 2012).

The scientists also used their method to investigate lingering questions about cholesterol absorption and how this process is related to the absorption of fatty acids. In the zebrafish, the researchers found that the presence of one type of fatty acid, oleic acid, is required for cholesterol uptake by enterocytes. Oleic acid also regulates the location within the enterocyte of the protein NPC1L1, which is integral to the process by which newly absorbed cholesterol combines with proteins to form lipoproteins. The intracellular location of NPC1L1 is fundamental to its role in promoting cholesterol uptake.

This study provides insights into the relationship between cholesterol and fatty acid metabolism that could have broad applications for human health. The researchers emphasize that their technique may be used in future studies to investigate other aspects of lipid metabolism as well.

\section{Kara Rosania}

\section{SOCIAL THERAPY, THE NEW ANTI-AGING BUZ7?}

Like many animals, honeybees (Apis mellifera) suffer from declining brain function as they age. The pace of this decline varies among individuals, partly because of variations in social behavior. Gro V. Amdam (Arizona State University, Tempe, and Norwegian University of Life Sciences, Aas) and colleagues recently took a closer look at the effects of social behavior on aging-related declines in brain function in honeybees. They found that such declines were specifically associated with certain social roles and, moreover, could be reversed by changes in social behavior. The results suggest that social interventions may be effective treatment strategies for age-related declines in brain function in humans.

Worker honeybees have various roles in the hive. Younger females (4-20 days old) typically remain in the hive, tending to the larvae and the queen, whereas older females (18-32 days old) typically leave the hive to forage for resources. Brain function, as measured by a sensory sensitivity test, remains fairly steady in the nurse bees but declines rapidly in foragers, who also age rapidly and die relatively quickly after switching roles. To find out whether this difference was reversible, Amdam's group removed the nurse bees from a nest. After several days of latency, some of the foragers took on nurse duties. About 10 days later, the foragers-turnednurses had regained some brain function (Exp. Gerontol. published online 21 May 2012; doi:10.1016/j.exger.2012.05.011). This recovery was positively associated with levels of cellular stress-response and maintenance proteins in the central brain.

The study results emphasize the value of honeybees as a model for aging research; provide evidence for the reversibility of learning deficits in aged honeybees; and indicate that this reversibility is related to mechanisms of cell stress response, maintenance and repair. Future studies in mammalian models may help to elucidate whether similar changes occur in these animals. Additionally, more research is needed to investigate how social influences, such as activities and relationships, affect the aging process overall.

Social interventions may offer a new avenue for treating age-related declines in brain function, including dementia. Effective treatments for maintaining brain function are highly sought-after by scientists and pharmaceutical companies. But developing a drug therapy requires extensive basic research and rigorous trials that can take many years to complete. "Maybe social interventions-changing how you deal with your surroundings-is something we can do today to help our brains stay younger," Amdam said in a press release. 